\title{
Correction to: Design of Data-Driven Visualization Teaching System for Preschool Basketball Courses
}

Yun-fei Qin, Lu-zhen Mo, and Can Chen

\author{
Correction to: \\ Chapter "Design of Data-Driven Visualization Teaching \\ System for Preschool Basketball Courses" \\ in: S. Liu et al. (Eds.): e-Learning, e-Education, \\ and Online Training, LNICST 340, \\ https://doi.org/10.1007/978-3-030-63955-6_25
}

In the version of this paper that was originally published the names of Y.-f. Qin and L.-z. Mo have been corrected to Yun-fei Qin and Lu-zhen Mo. 